\title{
Typical patterns of expiratory flow and carbon dioxide in mechanically ventilated patients with spontaneous breathing
}

\author{
S. E. Rees ${ }^{1}$ (D) S. Larraza ${ }^{1} \cdot$ N. Dey ${ }^{2} \cdot$ S. Spadaro ${ }^{3} \cdot$ J. B. Brohus ${ }^{4} \cdot$ \\ R. W. Winding ${ }^{2}$ C. A. Volta ${ }^{3}$ D. S. Karbing ${ }^{1}$
}

Received: 11 April 2016/Accepted: 20 June 2016/Published online: 25 June 2016

(C) Springer Science+Business Media Dordrecht 2016

\begin{abstract}
Incomplete expiration of tidal volume can lead to dynamic hyperinflation and auto-PEEP. Methods are available for assessing these, but are not appropriate for patients with respiratory muscle activity, as occurs in pressure support. Information may exist in expiratory flow and carbon dioxide measurements, which, when taken together, may help characterize dynamic hyperinflation. This paper postulates such patterns and investigates whether these can be seen systematically in data. Two variables are proposed summarizing the number of incomplete expirations quantified as a lack of return to zero flow in expiration (IncExp), and the end tidal $\mathrm{CO}_{2}$ variability (varETCO 2$)$, over 20 breaths. Using these variables, three patterns of ventilation are postulated: (a) few incomplete expirations $(\operatorname{IncExp}<2$ ) and small varETCO $\mathrm{O}_{2}$; (b) a variable number of incomplete expirations $(2 \leq \operatorname{IncExp} \leq 18)$ and large varETCO $\mathrm{C}_{2}$; and (c) a large number of incomplete expirations (IncExp > 18) and small varETCO ${ }_{2}$. IncExp and varETCO $\mathrm{CO}_{2}$ were calculated from data describing respiratory flow and $\mathrm{CO}_{2}$ signals in 11 patients mechanically ventilated at 5 levels of pressure support. Data analysis showed that the three patterns presented systematically in the data, with periods of
\end{abstract}

$\triangle$ S. E. Rees

sr@hst.aau.dk

1 Respiratory and Critical Care Group (rcare), Department of Health Science and Technology, Aalborg University, Fredrik Bajersvej 7E, 9220 Aalborg Øst, Denmark

2 Department of Anaesthesia and Intensive Care, Regions Hospital Herning, Herning, Denmark

3 Section of Anaesthesia and Intensive Care, Department of Morphology, Experimental Medicine and Surgery, Arcispedale Sant'Anna, University of Ferrara, Ferrara, Italy

4 Mermaid Care A/S, Nørresundby, Denmark
IncExp $<2$ or IncExp $>18$ having significantly lower variability in end-tidal $\mathrm{CO}_{2}$ than periods with $2 \leq \mathrm{In}-$ cExp $\leq 18 \quad(p<0.05)$. It was also shown that sudden change in IncExp from either IncExp $<2$ or IncExp $>18$ to $2 \leq$ IncExp $\leq 18$ results in significant, rapid, change in the variability of end-tidal $\mathrm{CO}_{2} p<0.05$. This study illustrates that systematic patterns of expiratory flow and end-tidal $\mathrm{CO}_{2}$ are present in patients in supported mechanical ventilation, and that changes between these patterns can be identified. Further studies are required to see if these patterns characterize dynamic hyperinflation. If so, then their combination may provide a useful addition to understanding the patient at the bedside.

Keywords Pressure support · Capnography · Expiratory flow $\cdot$ Dynamic hyperinflation

\section{Introduction}

Pressure support is often provided to mechanically ventilated patients with spontaneous breathing. Selection of the correct level of support is important. Inappropriate over- or under-support may result in inadequate time for complete expiration of inspiratory volume, resulting in dynamic hyperinflation or gas trapping, and the associated increase in pressure, known as autoPEEP (auto positive end expiratory pressure) or intrinsic PEEP [1-3].

Methods are available for measuring dynamic hyperinflation or autoPEEP. End expiratory pause or holds have been used in experimental studies [1-3]. Analysis of measurements taken during pause or hold require the absence of respiratory muscle activity and are therefore are not appropriate in pressure support ventilation. In clinical practice, the most routinely used method is to visually 
inspect whether the expiratory flow profile returns to zero at the end of expiration [4-6]. Simple, bedside interpretation of the expiratory flow profile, and its automated analysis [5], requires that these profiles are similar from breath to breath. In patients with spontaneous breathing activity, this is not the case making this method inappropriate for use in pressure support.

The expiratory $\mathrm{CO}_{2}$ profile may aid in the understanding of the breath to breath variability of expiratory flow and hence in understanding conditions such as dynamic hyperinflation. The expiratory $\mathrm{CO}_{2}$ profile is likely to reflect similar information as the expiratory flow signal. An incomplete expiration, characterized by expiratory flow not returning to zero, may be seen as early termination of phase III of the expiratory $\mathrm{CO}_{2}$ signal and, as a consequence, a relatively low end-tidal $\mathrm{CO}_{2}\left(\mathrm{PeTCO}_{2}\right)$ level. In contrast, a complete expiration, characterized by expiratory flow returning to zero, would result in $\mathrm{PETCO}_{2}$ estimated from later in the expiratory $\mathrm{CO}_{2}$ signal, and a relatively high $\mathrm{PETCO}_{2}$. A variable pattern of breathing, including flow profiles showing complete and incomplete breaths should therefore present with great variability in $\mathrm{PETCO}_{2}$.

This paper investigates whether patterns of expiratory flow and capnography exist in mechanically ventilated patients with spontaneous breathing. Due to the breath by breath variability in flow and expiratory $\mathrm{CO}_{2}$ seen in these patients, variables are defined averaging information from the flow and $\mathrm{CO}_{2}$ signals over a number of breaths, and patterns investigated in these summary variables. Several patterns are postulated based on patient examples, and it is investigated whether these exist systematically in patient data. Identification of such patterns may provide a useful source of information for understanding the degree and nature of dynamic hyperinflation at the bedside.

\section{Methods}

\subsection{Postulated patterns of expiratory flow and $\mathrm{CO}_{2}$ variability}

Figure 1 illustrates segments of data taken from three of the patients included in this study, with each column reflecting a different patient all of which were mechanically ventilated on pressure support ventilation. The first column of Fig. 1 illustrates data showing a respiratory pattern where flow returns to zero on all breaths (Fig. 1a), and the expiratory $\mathrm{CO}_{2}$ signal shows relatively well defined plateau values, with values of $\mathrm{PETCO}_{2}$ similar for each expiration (Fig. 1d). Both these signals would suggest that expiration is complete. The second column illustrates data showing a more irregular breathing pattern with flow returning to zero only periodically (Fig. 1b), with this due in part to ineffective respiratory efforts, and, no clear plateau being seen in the expiratory $\mathrm{CO}_{2}$ signal (Fig. 1e). This pattern suggests great variability in expiration and results in breath to breath variability in $\mathrm{PeTCO}_{2}$. The third column represents a regular breathing pattern, but rather different from column 1 . The expiratory flow signal does not return to zero (Fig. 1c) and there is no plateau in the expiratory $\mathrm{CO}_{2}$ signal (Fig. 1f), with very low $\mathrm{PeTCO}_{2}$ levels. In this patient all expirations are incomplete, but there is little breath to breath variability in $\mathrm{PeTCO}_{2}$.

These three patterns: Complete expirations and a stable $\mathrm{PETCO}_{2}$; intermittent incomplete expirations and variable $\mathrm{PETCO}_{2}$; and incomplete expirations and stable $\mathrm{PETCO}_{2}$; are visualized in plots of summary variables in Row 3 of Fig. 1 . The details of calculation of these summary variables are given in the section "Analysis of signals" at the end of the methods section. In each of these plots (Fig. 1g-i) the solid line illustrates the number of expirations that have not fully returned to zero flow in the last 20 breaths (IncExp). The dashed line illustrates the variability in $\mathrm{PETCO}_{2}$ over the last 20 breaths $\left.(\text { varETCO})_{2}\right)$. These summary variables describe the quite different patterns of ventilation seen in the three examples. For the first example (column 1) Fig. 1g illustrates that there are almost no breaths not returning to zero flow, and little $\mathrm{PETCO}_{2}$ variability. In the second example (column 2) Fig. 1h illustrates that there are some breaths not returning to zero flow, and substantial $\mathrm{PeTCO}_{2}$ variability. In the third example (column 3) Fig. 1i illustrates that breaths do not return to zero flow, but that there is little $\mathrm{PeTCO}_{2}$ variability.

It could be postulated that the three patterns illustrated in Fig. 1 represent general patterns seen in mechanically ventilated patients with spontaneous breathing effort, and that these patterns could be identified using the summary variables. This paper investigates whether these patterns, and changes in these patterns, can be identified in bedside measurements of respiratory $\mathrm{CO}_{2}$ and flow.

\subsection{Patient data}

Data describing respiratory flow and $\mathrm{CO}_{2}$ levels were analyzed from 11 patients on pressure support ventilation. Data were collected with ethical approval from the Ethics Committee of Mid-Jutland, Denmark. Informed written and oral consent was obtained from patients or relatives and, in the case of the latter, patients' general practitioner as required by Danish law. Parts of the data describing these patients have been published previously [7], and only previously unpublished data specifically related to continuous monitoring of flow and $\mathrm{CO}_{2}$ are considered further here.

Full details of the clinical protocol are given in [7], with only details relevant for this manuscript presented here. As 

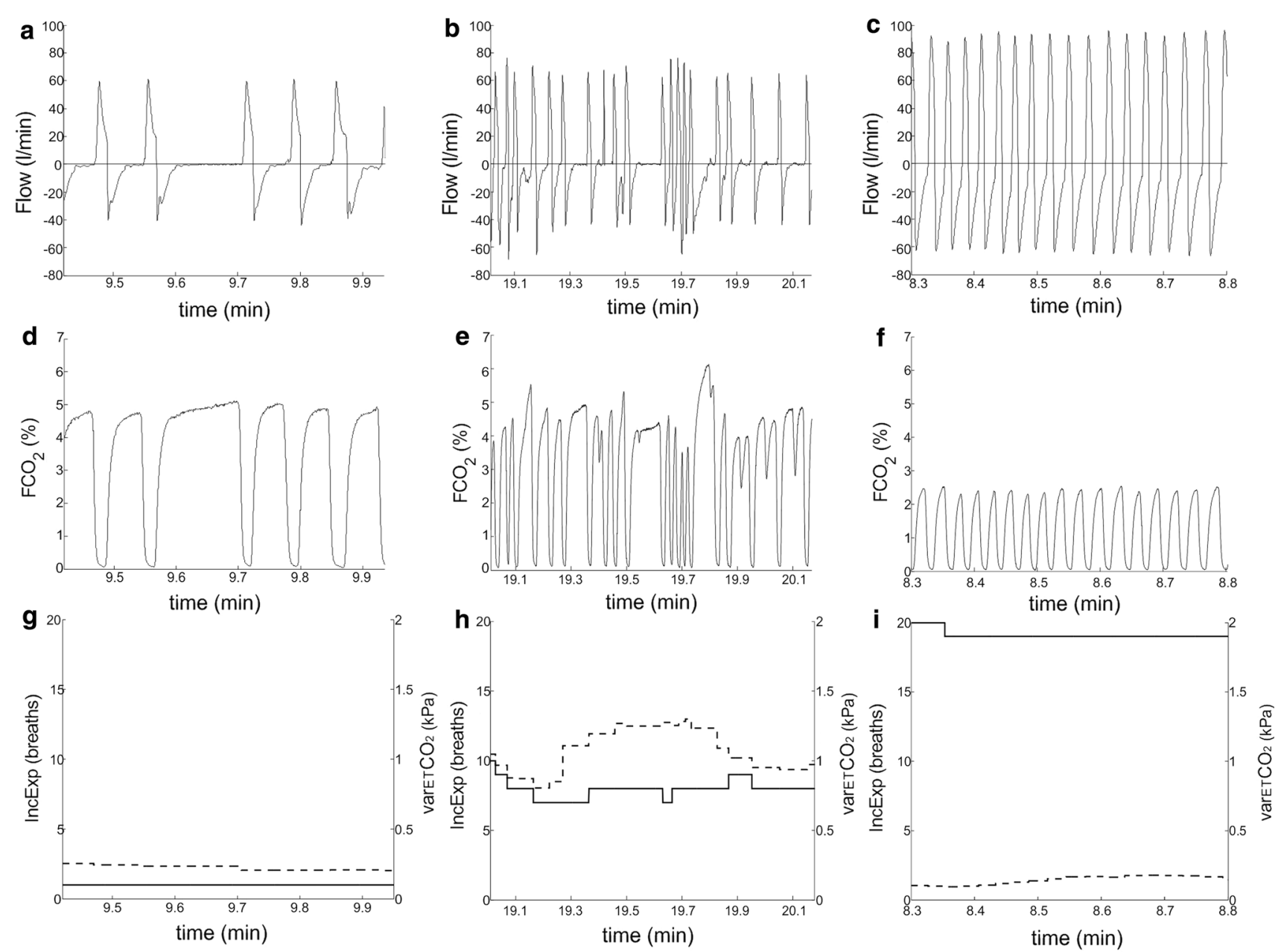

Fig. 1 Respiratory patterns in 3 data sets: a, d, $\mathbf{g}-$ few incomplete expirations and little $\mathrm{CO}_{2}$ variability; $\mathbf{b}, \mathbf{e}, \mathbf{h}-$ some incomplete expirations and $\mathrm{CO}_{2}$ variability; $\mathbf{c}, \mathbf{f}, \mathbf{i}-$ incomplete expirations and little $\mathrm{CO}_{2}$ variability. For subplots $\mathbf{g}, \mathbf{h}$, $\mathbf{i}$ solid lines indicate the number of incomplete expirations (IncExp), and dashed lines the endtidal $\mathrm{CO}_{2}$ variability (vareTCO${ }_{2}$ ), in the last 20 breaths described previously, all patients were older than 18 years, intubated, and ventilated in pressure support ventilation. Patients were excluded if, at the time of inclusion, dynamic lung compliance was $<30 \mathrm{ml} / \mathrm{cmH}_{2} \mathrm{O}$, positive end-expiratory pressure was $\geq 10 \mathrm{cmH}_{2} \mathrm{O}$, systolic blood pressure was $<90 \mathrm{mmHg}$ with vasopressor, $\mathrm{PaCO}_{2}$ was greater than $8.5 \mathrm{kPa}$, if the patient was previously diagnosed with chronic obstructive pulmonary disease (COPD) or in the absence of arterial catheter for blood sampling. The exclusion of patients with COPD is addressed further in the discussion.

Patients were ventilated using continuous positive airway pressure-assisted spontaneous breathing ventilation mode (Evita XL; Dräger Medical, Lübeck, Germany). This mode is pressure controlled, flow cycled, patient triggered, and pressure supported [8], i.e. pressure support (PS). Trigger sensitivity was set to $5 \mathrm{l} / \mathrm{min}$ in accordance with local clinical practice, ramp time was adjusted to achieve an inspiratory flow $<60 \mathrm{l} / \mathrm{min}$, cycling off criterion was set to $25 \%$ of the peak inspiratory flow, apnea ventilation frequency was set to 5 per min, and automatic tube compensation was turned off. Pressure and flow profiles and airway concentration of $\mathrm{O}_{2}$ and $\mathrm{CO}_{2}$ were measured continuously (CARESCAPE; GE Healthcare, Helsinki, Finland).

As described previously [7], patients were subjected to a maximum of 5 PS step changes of $2 \mathrm{cmH}_{2} \mathrm{O}$ each, beginning with reduction of PS from initial clinical conditions. Each PS level was maintained for $15 \mathrm{~min}$. Pressure support was further reduced only if $\mathrm{pH}$ in arterial blood was $>7.3$ and respiratory frequency $<30$ per min, if $<5$ PS steps had been performed, and if PS was greater than $0 \mathrm{cmH}_{2} \mathrm{O}$. In case of completing the 5 PS step modifications solely with PS reduction, the protocol concluded with resetting the ventilator to initial clinical conditions. Otherwise, PS was increased to the baseline level and subsequently increased by $2 \mathrm{cmH}_{2} \mathrm{O}$ steps. Each PS level was maintained for 15 min. Pressure support was further increased only if tidal 
volume (VT) was $<8 \mathrm{~mL} / \mathrm{kg}$, if peak inspiratory pressure (PIP) was $<30 \mathrm{cmH}_{2} \mathrm{O}$, and if $<5$ PS step changes had been performed. After completion of the 5 PS step changes or if it was not possible to further increase PS, the protocol concluded by resetting the ventilator to initial clinical conditions.

\subsection{Data analysis}

The respiratory flow signals of all 11 patients were analysed to determine the two summary variables describing the number of incomplete expirations (IncExp) and variability of end tidal $\left.\mathrm{CO}_{2}(\text { vareTCO})_{2}\right)$ over 20 breaths. The calculation of these variables is described in the section "Analysis of signals".

Values of IncExp and varETCO $\mathrm{CO}_{2}$ were then analysed to evaluate two research questions.

\subsubsection{Are the three patterns illustrated in Fig. 1 present in the complete data set?}

A breath by breath classification of all patient data was performed, such that sets of data including 20 consecutive breaths were assigned to one of 3 groups representing the three patterns of ventilation seen in Fig. 1, according to IncExp. These were: $\operatorname{IncExp}<2 ; 2 \leq \operatorname{IncExp} \leq 18$; and IncExp $>18$. For all data in each of these groups, the median and range were calculated for varETCO $\mathrm{C}_{2}$. Box plots were used to visualize the value of vareTCO ${ }_{2}$ for each of the groups. A Wilcoxon rank sum test was used to compare values of varETCO $\mathrm{C}_{2}$ in each of the groups. A $p$ value of $<0.05$ was used to signify statistical significance.

\subsubsection{Are sudden changes in flow consistent with changes in the variability of end tidal $\mathrm{CO}_{2}$ ?}

For each patient, data were analyzed to identify situations where IncExp changed, i.e. where the patient's pattern of breathing switched between the three patterns shown in Fig. 1. Change in IncExp was defined as the value of IncExp moving between: (1) IncExp from $<2$ to $>18$; (2) IncExp from $<2$ to 2-18; (3) IncExp from 2-18 to $<2$; (4) IncExp from $2-18$ to $>18$ to; (5) IncExp from $>18$ to $2-18$; and (6) IncExp from $>18$ to $<2$. For each of these six changes the value of the median and range was calculated for the varETCO $\mathrm{O}_{2}$ immediately prior to and then following the change. Box plots were used to visualize the value of varETCO ${ }_{2}$ on changing classification. A paired MannWhitney test was used to compare median $\operatorname{varETCO} \mathrm{CO}_{2}$ variation before and after the changes. A $p$ value of $<0.05$ was used to signify statistical significance.

To place these correlations in a clinical context, individual examples of flow, $\mathrm{CO}_{2}$, IncExp and varETCO $\mathrm{CO}_{2}$ were identified in the data set to describe typical scenarios of changes in respiratory pattern. These examples are illustrated in figures.

\subsection{Analysis of signals}

\subsubsection{Analysis of flow signal}

Figure 2 illustrates analysis of the expiratory flow profile. For each breath, the end of expiration was identified as a change in flow from negative to positive. To prevent erroneous identification, end expiration was confirmed by identifying an inspiratory volume of $50 \mathrm{ml}$ subsequently. This definition of end expiration was used in both flow and $\mathrm{CO}_{2}$ analysis.

The expiratory flow signal was integrated to calculate the ventilation volume in the last $0.5 \mathrm{~s}$ of expiration, as illustrated in Fig. 2. We postulated that significant volume in this period was consistent with an incomplete expiration. This volume can be caused by numerous physiological conditions including; a slow emptying of the lungs due to high resistance; a reduced expiratory driving pressure; or due to the contraction of inspiratory muscles during the expiratory phase, i.e. a wasted effort. In the latter case inspiratory muscle contraction may slow expiratory flow, despite not being sufficient to trigger the ventilator. We classified the breath to be incomplete if this volume exceeded $35 \mathrm{ml}$. The value of the time window of $0.5 \mathrm{~s}$ was selected to allow this analysis even at high respiratory frequency. The value of $35 \mathrm{ml}$ was an arbitrary selection. To confirm that $0.5 \mathrm{~s}$ time window and $35 \mathrm{ml}$ volume limit could identify incomplete expiration, these values were used to analyse flow data collected from an average of 8500 breaths per patient in data from 4 previously studied patients, outside this study. These patients were ventilated with PS and had expiratory flow limitation (EFL) confirmed with an expiratory PEEP reduction, as described previously [9]. Despite EFL, two of these patients had an insignificant dynamic intrinsic $\mathrm{PEEP}<1 \mathrm{cmH}_{2} \mathrm{O}$, as

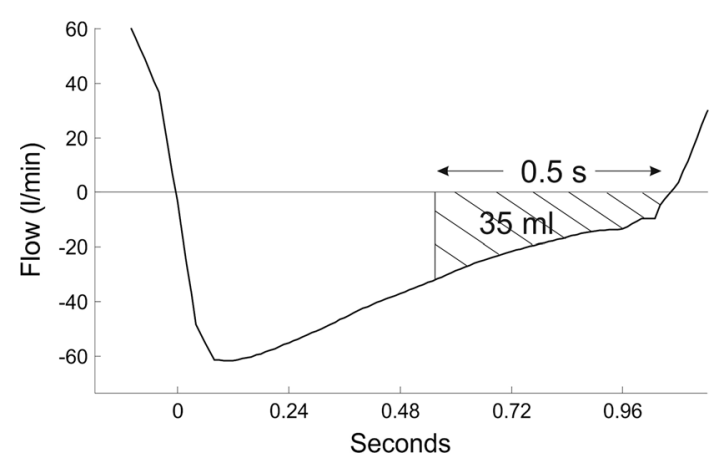

Fig. 2 Analysis of incomplete expiration identified as $>35 \mathrm{ml}$ of volume in the last $0.5 \mathrm{~s}$ of expiration 
measured with esophageal pressure. Analysis of these data using a $0.5 \mathrm{~s}$ time window and a $35 \mathrm{ml}$ volume limit showed that for the two patients without dynamic intrinsic PEEP $90 \%$ of breaths were classified as complete, with $10 \%$ classified as complete in the two patients with significant dynamic intrinsic PEEP.

IncExp was then calculated as a rolling average of the number of expirations classified as incomplete in the last 20 breaths, with 20 breaths selected to allow a time window of about 0.5-2 min. The value of IncExp was used to categorize all data into groups. We hypothesized that the extremes of the range of IncExp could be set at $10 \%$ limits, giving categories in the ranges $<2$ expirations incomplete; $2-18$ expirations incomplete, and $>18$ expirations incomplete, all in the previous 20 breaths.

\subsubsection{Analysis of the capnography signal}

$\mathrm{PeTCO}_{2}$ values were calculated as the maximal $\mathrm{CO}_{2}$ during the expiratory period. Maximal values were chosen, rather than end expiratory values, so as to best to approximate the alveolar partial pressure and prevent error due to factors such as re-breathing due to inspiratory muscle effort during the expiratory phase.

To reflect the breath by breath variability in $\mathrm{PeTCO}_{2}$ a rolling average was calculated as two times the standard deviation of $\mathrm{PETCO}_{2}$ over the previous 20 breaths $(\operatorname{varETCO})_{2}$.

\section{Results}

Figure 3 illustrates box plots describing vareTCO $\mathrm{O}_{2}$ for the data divided into the three groups. 223 data sets were present describing 20 breath periods of breathing where IncExp $<2$,

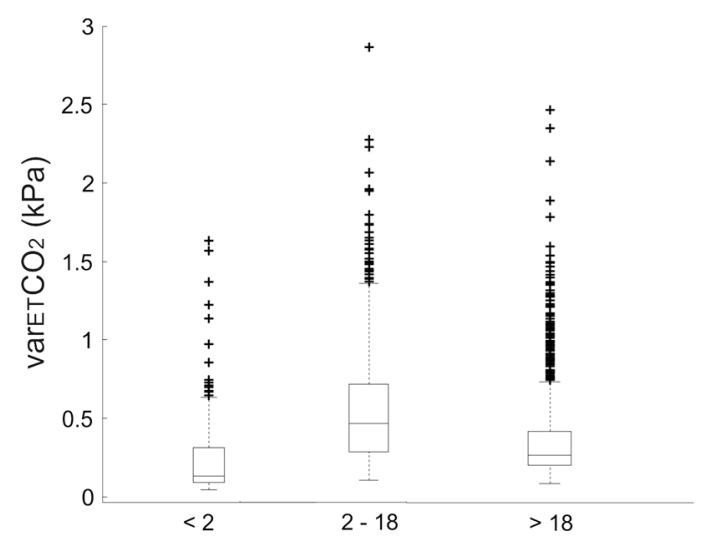

Fig. 3 End-tidal $\mathrm{CO}_{2}$ variability (varETCO 2 ) for breath data from all patients classified according to $\operatorname{IncExp}<2,2 \leq \operatorname{IncExp} \leq 18$ and IncExp $>18$. Box-plots indicate median, 25 and 75 percentile (box), and range (whiskers) excluding outliers. Points are considered outliers (crosses) if outside 1.5 times the upper and lower inter quartile ranges, respectively with the median varEtCO $2=0.13 \mathrm{kPa} .1149$ data sets were present describing 20 breath periods of breathing where $2 \leq \operatorname{IncExp} \leq 18$, with the median varETCO ${ }_{2}=0.44 \mathrm{kPa}$. 978 data sets were present describing 20 breath periods of breathing where IncExp $>18$, with the median varEtCO ${ }_{2}=0.19 \mathrm{kPa}$. The median varETCO $\mathrm{O}_{2}$ differed significantly $(p<0.05)$ between groups.

Figure 4 illustrates box plots describing the varETCO $\mathrm{CO}_{2}$ on change of IncExp classification. Box plots are shown in the figure for four of the six groups with only one data sample available for groups (1) and (6) and these groups not shown.

The first row of Fig. 4 illustrates varETCO $\mathrm{CO}_{2}$ on change of IncExp from $<2$ incomplete expirations in the previous 20 , to between 2 and 18 incomplete expirations in the last 20 $(n=45)$; and on change of IncExp from between 2 and 18 incomplete expirations in the last 20 , to $<2$ incomplete expirations in the previous $20(\mathrm{n}=46)$. In both cases, a switch between these groups is associated with a significant change in $\operatorname{vareTCO}_{2}(p<0.05)$, with the median of
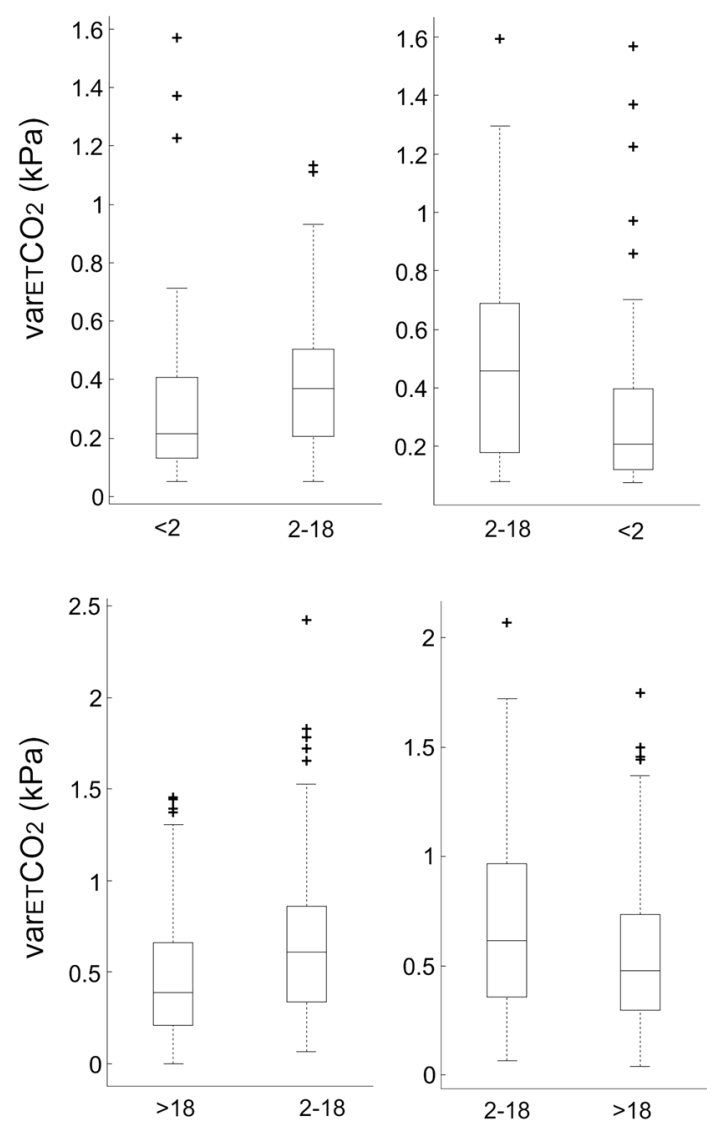

Fig. 4 End-tidal $\mathrm{CO}_{2}$ variability (varETCO 2 ) for data where the number of incomplete expirations in the last 20 changes from $<2$ to $2-18 ; 2-18$ to $<2 ;>18$ to $2-18$; and $2-18$ to $>18$. Box-plots indicate median, 25 and 75 percentile (box), and range (whiskers) excluding outliers. Points are considered outliers (crosses) if outside 1.5 times the upper and lower inter quartile ranges, respectively 
varETCO $\mathrm{CO}_{2}$ changing from $0.21 \mathrm{kPa}$ to $0.37 \mathrm{kPa}$ on IncExp change from $<2$ to $2-18$; and the median varETCO changing from 0.46 to $0.21 \mathrm{kPa}$ on IncExp change from $2-18$ to $<2$.

The second row of Fig. 4 illustrates varETCO${ }_{2}$ on change of IncExp from $>18$ incomplete expirations in the previous 20 , to between 2 and 18 incomplete expirations in the last 20 ( $\mathrm{n}=119)$; and on change of IncExp from between 2 and 18 incomplete expirations in the last 20 , to $>18$ incomplete expirations in the previous $20(n=114)$. In both cases, a switch between these groups is associated with a significant change in varETCO $\mathrm{C}_{2}(p<0.05)$, with the median of varETCO $\mathrm{C}_{2}$ changing from 0.39 to $0.61 \mathrm{kPa}$ on IncExp change from $>18$ to $2-18$; the median of varETCO $\mathrm{CO}_{2}$ changing from 0.61 to $0.48 \mathrm{kPa}$ on IncExp change from $2-18$ to $>18$.

\section{Typical scenarios of respiratory patterns}

In addition to the analysis of the flow and $\mathrm{CO}_{2}$ patterns seen in the group as a whole, several interesting patterns can be observed in the data. Four of these are presented here, with two illustrating the link between IncExp and varETCO $\mathrm{O}_{2}$ over time; and a further two providing examples of changes in these variables on changes in state.

\subsection{Examples illustrating the link between IncExp and vareTCO $\mathrm{CO}_{2}$ over time}

Figure 5 illustrates a plot of IncExp (black line) and varETCO $\mathrm{CO}_{2}$ (blue line) over the whole $210 \mathrm{~min}$ period of data collection in one of the patients in the study group.

This patient was ventilated with PS values ranging from 3 to $12 \mathrm{cmH}_{2} \mathrm{O}$. The patient had a respiratory frequency varying from 10 to 20 breaths per minute. The level of PS

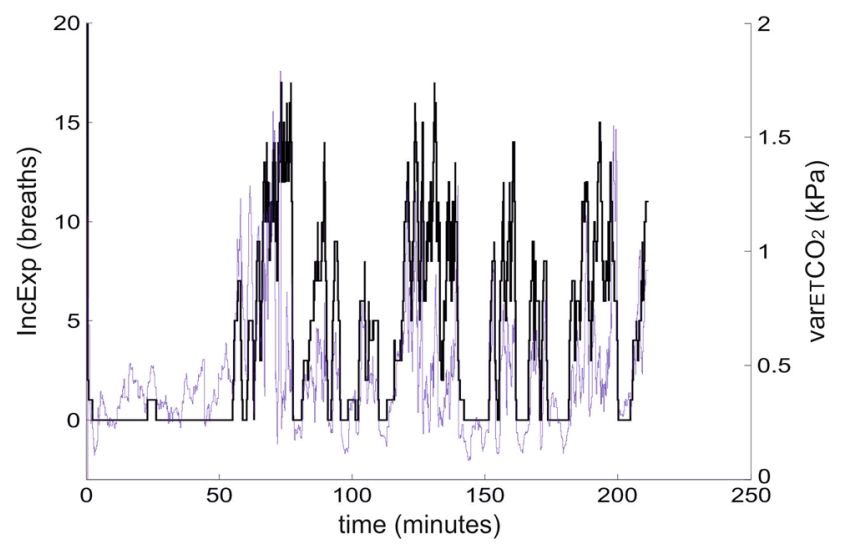

Fig. 5 The number of incomplete expirations (IncExp) in the last 20 (black line) and the end-tidal $\mathrm{CO}_{2}$ variability $\left(\right.$ vareTCO $\left.{ }_{2}\right)$ (blue line) for a patient monitored for $210 \mathrm{~min}$ was not correlated with either summary variable or respiratory frequency. IncExp and varETCO $\mathrm{CO}_{2}$ follow each other quite closely, illustrating periods of few incomplete expirations and little $\mathrm{CO}_{2}$ variability, and periods where both are increased.

Figure 6 illustrates a plot of IncExp (bold line) and varEtCO ${ }_{2}$ (thin line) over $130 \mathrm{~min}$ in a different patient from that shown in Fig. 5. This patient was ventilated with PS values ranging from 4 to $14 \mathrm{cmH}_{2} \mathrm{O}$ and had a respiratory frequency of around 40 breaths per minute for the complete duration. The level of PS was not correlated with either summary variable or respiratory frequency. The patient had a high value of IncExp, with the majority of expirations not reaching zero flow and a low value of varETCO $\mathrm{CO}_{2}$ It is interesting that sudden reduction in the number of breaths not reaching zero flow, i.e. a reduction in IncExp, is consistent with sudden increase in $\mathrm{CO}_{2}$ variability, with spikes in both signals occurring at the same time, as can be identified around 60, 90 and $120 \mathrm{~min}$.

\subsection{Examples of changes in patient state}

Figure 7 shows data from two patients illustrating continuous flow and $\mathrm{CO}_{2}$ signals, and values of IncExp and varETCO $\mathrm{C}_{2}$ over short periods of time. For each of these patients a region of data is focused on at the time where the patient changed state between the three classification groups.

The first column illustrates $7 \mathrm{~min}$ of data from a patient where most expirations were complete. Two short periods of high respiratory frequency can be seen in the flow signal (Fig. 7a) consistent with a short period of patient stress around 94 and $95.5 \mathrm{~min}$. An extra figure is shown zooming in on the flow signal of first of these (Fig. 7c). During the period including this higher frequency, expirations were incomplete and $\mathrm{PETCO}_{2}$ was very variable, as can be seen

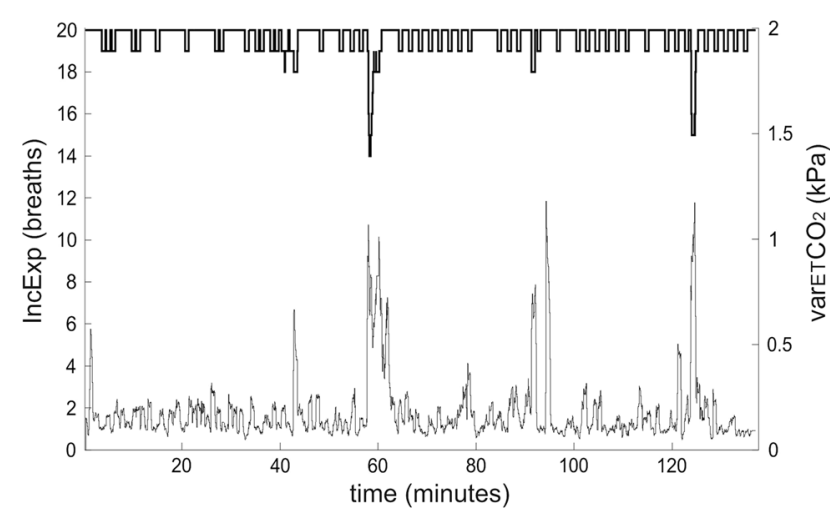

Fig. 6 The number of incomplete expirations in the last 20 breaths (IncExp) (bold line) and the end-tidal $\mathrm{CO}_{2}$ variability $\left(\right.$ varEtCO $\mathrm{C}_{2}$ ) (thin line) for a patient monitored for $140 \mathrm{~min}$ 
Fig. 7 Two examples (column 1 and column 2) illustrating flow $(\mathbf{a}, \mathbf{b}, \mathbf{c}), \mathrm{CO}_{2}(\mathbf{d}, \mathbf{e})$, and both IncExp and varETCO ${ }_{2}(\mathbf{f}$, g), in situations where the patients change state. In subplots $\mathbf{f}, \mathbf{g}$, bold lines indicate IncExp, thin lines indicate varETCO $\mathrm{C}_{2}$
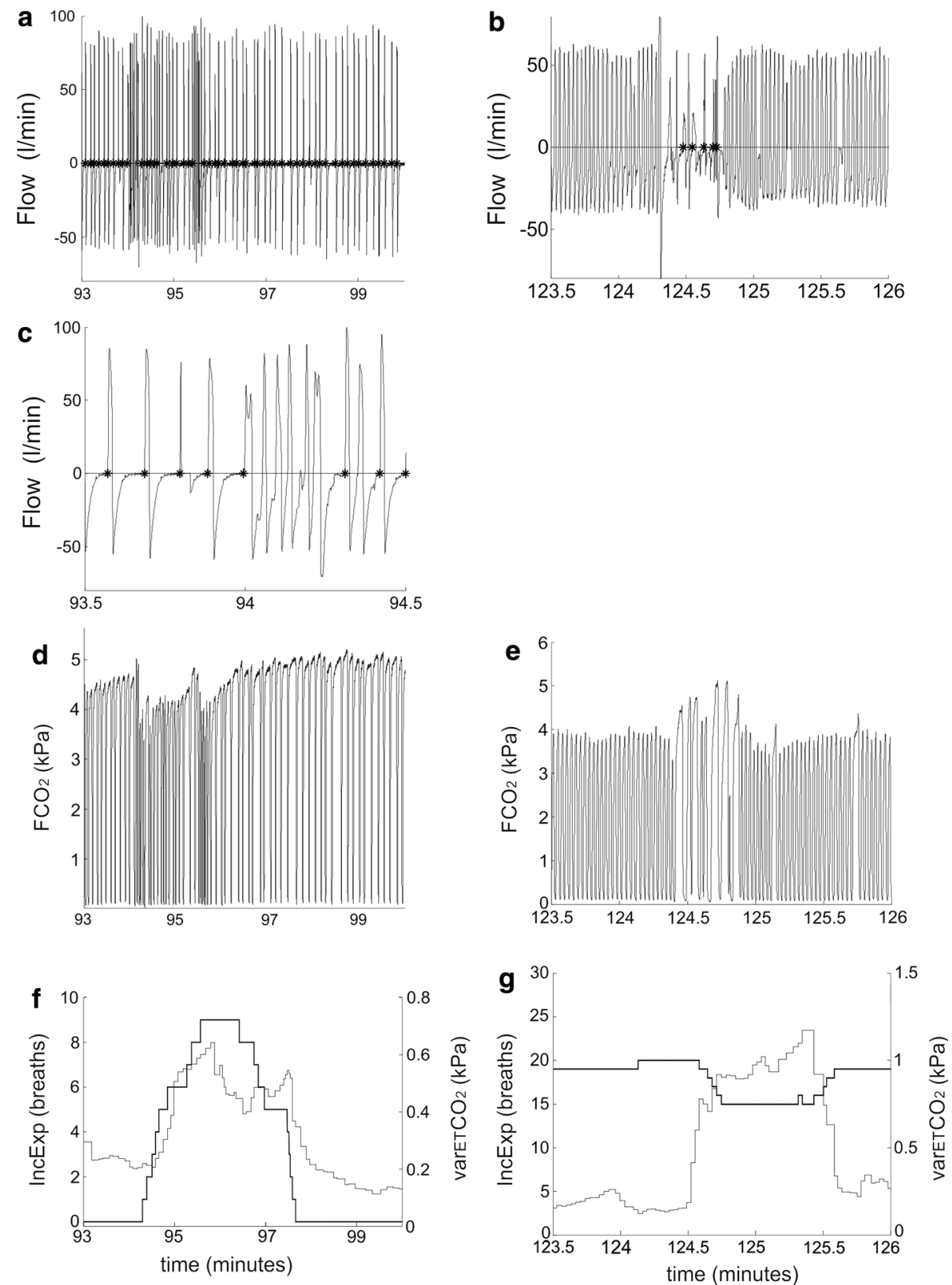

in the raw signals (Fig. 7a, c d) or the calculated values of IncExp and varETCO $\mathrm{O}_{2}$ (Fig. 7f). Values of IncExp and varETCO $\mathrm{C}_{2}$ stabilized as the patient returned to a more regular breathing pattern (Fig. 7f).

The second column illustrates about 3 min of data from a patient where expirations tended to be incomplete. A period of a number of consecutive breaths with complete expiration can be seen at around 124.5 min (Fig. 7b). During this period, $\mathrm{PeTCO}_{2}$ values increased (Fig. 7e), probably due to the washout of $\mathrm{CO}_{2}$ during complete expirations. This pattern is illustrated in values of IncExp and varETCO $\mathrm{C}_{2}$ shown in Fig. $7 \mathrm{~g}$. As the breathing pattern returns to predominantly incomplete expirations, end tidal
$\mathrm{CO}_{2}$ falls, and IncExp and varETCO $\mathrm{CO}_{2}$ return to high and low values, respectively (Fig. $7 \mathrm{~g}$ ).

\section{Discussion}

This paper has examined the relationship between the expiratory flow profile and expiratory $\mathrm{CO}_{2}$ signal of patients with spontaneous breathing ventilated in pressure support mode. It has been shown that three patterns exist in these data, such that few incomplete expirations is associated with a lower variability in end-tidal $\mathrm{CO}_{2}$, with this variability increasing with the number of incomplete 
expirations, until all but a few breaths are incomplete in which case end-tidal $\mathrm{CO}_{2}$ variability is again low.

In addition, it has been shown that when a patient changes flow pattern, by increasing or decreasing the number of incomplete breaths, then immediate changes in end-tidal $\mathrm{CO}_{2}$ variability can be seen, in line with the three postulated groups, illustrating that changes between the patterns can be identified.

The fact that these associations between number of incomplete expirations and variability in end tidal $\mathrm{CO}_{2}$ are present is promising. It may be the case that the pattern of few incomplete expirations and little $\mathrm{CO}_{2}$ variability represents a pattern where no gas trapping exists. It might also be the case that the pattern of almost all incomplete expirations and little $\mathrm{CO}_{2}$ variability indicates situations of gas trapping. Perhaps most interesting is the large variability seen in the middle range. It is possible that the periods of variability of flow and end tidal $\mathrm{CO}_{2}$ signal represent a pattern of "cyclic dynamic hyperinflation" where the autoPEEP generated by several incomplete expirations provides sufficient expiratory driving pressure to overcome resistance, enabling a full expiration and the resulting higher end tidal $\mathrm{CO}_{2}$. This pattern of autoPEEP build up and release would be consistent with a highly variable endtidal $\mathrm{CO}_{2}$, as seen in these data. If these patterns are descriptive of these physiological situations, then identification of changes between the three classifications might be important, and it is encouraging that significant differences in end-tidal $\mathrm{CO}_{2}$ variability can be seen across the groups as patients change state. The longer complete expirations being consistent with a later phase III value of end-tidal $\mathrm{CO}_{2}$ could also explain why maximal end-tidal values of $\mathrm{CO}_{2}$ provide an improved correlation with values of arterial $\mathrm{PaCO}_{2}$ as shown recently by Galia et al. [10].

The purpose of this paper has been to see if the postulated patterns of data exist systematically. This has been shown, but several limitations exist in the study and analysis presented here. It has not been shown that these patterns necessarily correlate with the presence or absence of dynamic hyperinflation or autoPEEP. To do so would require similar analysis but with simultaneous measurement of esophageal pressure. In addition, it may be the case that different flow and $\mathrm{CO}_{2}$ variables to InExp and varETCO ${ }_{2}$ provide similar patterns. IncExp was selected as analysis of the flow signal's return to zero is the most common method for identifying dynamic hyperinflation or autoPEEP [4-6]. The averaging over 20 breaths was selected so as to reflect the variability seen in patients with spontaneous breathing activity. Twenty breaths was selected so as to allow the analysis window to range from about $0.5-2 \mathrm{~min}$, this balancing the need for an index which provided adequate averaging but remained responsive to patient changes. varETCO ${ }_{2}$ was selected following visual inspection of all curves. It is possible that other variables, such as the length of the $\mathrm{CO}_{2}$ expirogram, would provide a similar description of the incompleteness of the breath and no systematic analysis of all possible variables has been conducted. In our opinion, the $\mathrm{CO}_{2}$ signal best describing the completeness of expiration is most likely $\mathrm{PeTCO}_{2}$, with complete expirations resulting in $\mathrm{PETCO}_{2}$ best approximating values from alveolar with the highest expiratory time constants, and variation in $\mathrm{PETCO}_{2}$ describing an variation in the patient's ability to expire from these alveolar compartments. A further limitation is that the effects of abnormal expiratory patterns such as ineffective inspiratory effort or expiratory flow limitation have not been investigated, apart from verifying that the flow analysis presented here is consistent with the data from four patients with EFL. Ineffective respiratory effort generates instability in the expiratory flow and $\mathrm{CO}_{2}$ signal, which would increase the calculated number of incomplete breaths and $\mathrm{CO}_{2}$ variability. As ineffective respiratory effort is associated with dynamic hyperinflation [11], these increases would seem reasonable. In addition, it was assumed that the appropriate threshold for the classification of the three groups was as the $10 \%$ limits of the number of incomplete expirations in the last 20 breaths. These limits were arbitrary, but if esophageal measurements were taken, and dynamic hyperinflation identified, then values for these thresholds and approximations could be tailored to reflect these measurements. Regardless of these approximations, it is clear from this analysis that patterns exist in the expiratory flow and capnography signal that may help understanding patient state.

The patients included in this study represented a broad range of primary diagnoses including post-operative sepsis, acute lung injury, aspiration pneumonia and cardiac arrest [7]. Patients with COPD were excluded from this study. For evaluating the three different postulated patterns this may be seen as an advantage. It has previously been shown that all patients mechanically ventilated and with COPD have intrinsic PEEP [12] and may therefore be relatively homogenous in terms of the patterns presented here. While investigation of these patterns in COPD is necessary, the patient group studied here, with large changes in pressure support, is probably most likely to show the greatest heterogeneity in these patterns, a postulate substantiated by the large differences seen in patients as illustrated in Figs. 5 and 6 . The patients included here were also ventilated at a wide range of settings with pressure support levels ranging from 3 to $20 \mathrm{cmH}_{2} \mathrm{O}$, resulting in maximal inspiratory pressures from 9 to $27 \mathrm{cmH}_{2} \mathrm{O}$ and respiratory frequency from 9 to 50 breaths/min [7]. PEEP levels were kept constant, with only a moderate range across patients, from 5 to $9 \mathrm{cmH}_{2} \mathrm{O}$. Investigating changes in these patterns with varying the settings of PEEP could be important. 
Patients with dynamic hyperinflation due to expiratory flow limitation are likely to respond differently to changes in PEEP settings than those where dynamic hyperinflation is due to "ohmic" resistance, as described previously [1]. The patterns of response to changes in PEEP settings may therefore look very different in these two groups.

If these patterns describing respiratory flow and $\mathrm{CO}_{2}$ variability could be shown to correlate with the presence of dynamic hyperinflation then they may be useful in bedside monitoring. Current methods for identifying dynamic hyperinflation involve maneuvers such as airway occlusion end expiratory pause, and for proper interpretation require the measurement of esophageal pressure [1-3]. Less invasive methods for identifying dynamic hyperinflation have been explored, these including the identification of pulsos paradox in arterial blood pressure waveforms [13, 14] or using measurement of the negative pressure generated during the first $100 \mathrm{~ms}$ of an occluded inspiration $\left(\mathrm{P}_{0.1}\right)$ as means to evaluate the change in work of breathing due to changes in dynamic hyperinflation on varying PEEP [15]. Only Stentz, Arnold and colleagues have previously evaluated the capnography signal in relation to changes in lung volume induced with modification in ventilator settings $[16,17]$. These authors found that useful information was present in the signal, with a single breath analysis of the phase III slope of the capnography signal correlating with lung volume on changing PEEP. These studies were, however, performed in control ventilation mode, where large breath-by breath variability in expiratory flow and $\mathrm{CO}_{2}$ signal seen in the data presented here is probably absent. Indeed, for the data analysed here, a phase III signal was not always present.

In summary, the data presented here show a clear role for the combination of signals describing the number of incomplete expirations and the end tidal $\mathrm{CO}_{2}$ variability, with systematic patterns presenting in these data sets. Further studies are required to see if they characterize dynamic hyperinflation. If so, then their combination may provide a useful addition to understanding patient physiology at the bedside.

\section{Compliance with ethical standards}

Conflict of interest S. E. Rees, S. Larraza and D. S. Karbing have applied for a patent based on the method presented here. Ownership of the patent is with Mermaid Care A/S. S. E. Rees and D. S. Karbing are minor shareholders, and S. E. Rees is a board member, of Mermaid Care.

\section{References}

1. Laghi F, Goyal A. Auto-PEEP in respiratory failure. Minerva Anestesiol. 2012;78:201-21.

2. Blanch L, Bernabé F, Lucangelo U. Measurement of air trapping, intrinsic positive end-expiratory pressure, and dynamic inflation in mechanically ventilated patients. Respir Care. 2005;50:110-24.

3. Marini JM. Dynamic hyperinflation and auto-positive end-expiratory pressure: lessons learned over 30 years. Am J Respir Crit Care Med. 2011;184:756-62.

4. Dhand R. Ventilator graphics and respiratory mechanics in the patient with obstructive lung disease. Respir Care. 2005;50(2):246-61.

5. Nguyen QT, Pastor D, L'Her E. automatic detection of autoPEEP during controlled mechanical ventilation. Biomed Eng Online. 2012;11:32.

6. Lourens MS, van den Berg B, Aerts JGJV, Verbraak AFM, Hoogsteden HC, Bogaard JM. Expiratory time constants in mechanically ventilated patients with and without COPD. Intensive Care Med. 2000;26:1612-8.

7. Larraza S, Dey N, Karbing DS, Jensen JB, Nygaard M, Winding $\mathrm{R}$, Rees SE. A mathematical model approach quantifying patients' response to changes in mechanical ventilation: evaluation in pressure support. J Crit Care. 2015;30(5):1008-15.

8. Deden K. Ventilation modes in intensive care. Lübeck: DrägerwerkAG \& Co.; 2010.

9. Marangoni E, Alvisi V, Ragazzi R, Mojoli F, Alvisi R, Caramori G, Astolfi L, Volta CA. Respiratory mechanics at different PEEP levels during general anesthesia in the elderly: a pilot study. Minerva Anestesiol. 2012;78:1205-14.

10. Galia F, Brimioulle S, Bonnier F, Vandenbergen N, Dojat M, Vincent J-L, Brochard LJ. Use of maximal end-tidal $\mathrm{CO}_{2}$ values to improve end-tidal $\mathrm{CO}_{2}$ monitoring accuracy. Respir Care. 2011;56(3):278-83.

11. Rossi A, Ganassini A, Polese G, Grassi V. Pulmonary hyperinflation and ventilator-dependent patients. Eur Respir J. 1997;10(7):1663-74.

12. Bernasconi M, Ploysongsang Y, Gottfried SB, Milic-Emili J, Rossi A. Respiratory compliance and resistance in mechanically ventilated patients with acute respiratory failure. Intensive Care Med. 1988;14(5):547-53.

13. Perel A, Segal E. Systolic pressure variation-a way to recognize dynamic hyperinflation. Br J Anaesth. 1996;76:168-9.

14. Hartert TV, Wheeler AP, Sheller JR. Use of pulse oximetry to recognize severity of airflow obstruction in obstructive airway disease. Chest. 1999;115:475-81.

15. Mancebo J, Albaladejo P, Touchard D, Bak E, Subirana M, Lemaire F, Harf A, Brochard L. Airway occlusion pressure to titrate positive end-expiratory pressure in patients with dynamic hyperinflation. Anesthesiology. 2000;93(1):81-90.

16. Stenz RI, Grenier B, Thompson JE, Arnold JH. Single-breath $\mathrm{CO}_{2}$ analysis as a predictor of lung volume in a healthy animal model during controlled ventilation. Crit Care Med. 1998;26(8):1409-13.

17. Arnold JH, Stenz RI, Grenier B, Thompson JE. Single-breath $\mathrm{CO}_{2}$ analysis as a predictor of lung volume change in a model of acute lung injury. Crit Care Med. 2000;28(3):760-4. 